\title{
Reliability of Buried Pipes in Heterogeneous Soil Subjected to Seismic Loads
}

\author{
Hichem Benzeguir \\ Department of Civil Engineering \\ University Djillali Liabes of Sidi Bel Abbes \\ Sidi Bel Abbes, Algeria \\ h_benzeguir@yahoo.fr \\ Djamel Nedjar \\ Department of Civil Engineering \\ University of Sciences and Technology of Oran \\ Oran, Algeria \\ djamel_nedjar@yahoo.fr
}

\author{
Sidi Mohamed Elachachi \\ Civil and Environmental Engineering Department \\ University of Bordeaux \\ Talence, France \\ sm_elachachi@yahoo.fr

\section{Mohamed Bensafi} \\ Department of Civil Engineering \\ University of Sciences and Technology of Oran \\ Oran, Algeria \\ moha_bensafi@yahoo.fr
}

\begin{abstract}
Dysfunctions and failures of buried pipe networks, like sewer networks, are studied in this paper from the point of view of structural reliability and heterogeneity of geotechnical conditions in the longitudinal direction. Combined soil spatial variability and Peak Ground Acceleration (PGA) induce stresses and displacements. A model has been developed within the frame of geostatistics and a mechanical description of the soil-structure interaction of a set of buried pipes with connections resting on the soil by a two-parameter model (Pasternak model). Structural reliability analysis is performed considering two limit states: Serviceability Limit State (SLS), related to large "counter slope" in a given pipe, and Ultimate Limit State (ULS), corresponding to bending moment.
\end{abstract}

Keywords-reliability index; soil-structure interaction; spatial variability; $S L S$; $U L S$; seismic action

\section{INTRODUCTION}

Pipes that carry various substances need to be designed in a way capable of reducing the damage caused by ground displacements induced by earthquakes. The geoenvironmental effect has attracted concern on the performance of the buried pipes because of the associated hazards [1]. Most buried pipelines in seismic areas have sustained substantial damage in the past due to earthquake events [2]. Dysfunctions and failures of buried pipe networks, like sewer networks, are mainly caused by the heterogeneity of geotechnical conditions in the longitudinal direction and of the applied (seismic) action. Combined soil defects (differential settlements along the pipe, landslides, voids surrounding the pipe, etc.) and Peak Ground Acceleration (PGA) induce stresses (which lead to an Ultimate Limit State-ULS) and displacements (which constitute a violation of the Serviceability Limit State-SLS). It is worth noting that the influence of the variability of the soil is not reflected in current European standards [3]. Authors in [4] presented a comprehensive literature review on the seismic behavior of buried pipelines and underground structures summarizing the recent research. Structural response to ground motion during earthquake cannot be accurately predicted because of the complexity of the structural properties and ground motion parameters [5]. In this paper, the response of buried pipe is investigated considering the seismic excitation by selecting real earthquake data.

\section{SOIL PIPE SYSTEM MODELING}

\section{A. Pasternak Model}

Among the models that describe the behavior of a beam resting on a soil and their interaction, the Pasternak model [6] attracts the most interest. In the soil-pipe interaction, the soil opposes on the components of a sewer network (pipes) a distributed force $R(x)$ (in $\mathrm{N} / \mathrm{m}$ ) given by (1):

$$
R(x)=p(x) \cdot D_{\text {ext }}
$$

where $p(x)$ is the stress under the pipe $(\mathrm{Pa})$, and $D_{\text {ext }}$ the external diameter of the pipe $(\mathrm{m})$. According to the Pasternak model the stress is expressed as:

$$
p(x)=k_{w} \cdot w(x)-k_{s} \frac{d^{2} w}{d x^{2}}
$$

where $k_{w}$ is the coefficient of subgrade reaction (or Winkler coefficient in $\mathrm{N} / \mathrm{m}^{3}$ or $\left.\mathrm{Pa} / \mathrm{m}\right), k_{s}$ is the shear coefficient $(\mathrm{N} / \mathrm{m})$ and $w(x)$ the vertical pipe displacement (and thus the settlement of the soil).

Pasternak's idealization considers the soil as being a system of identical but mutually independent, closely spaced, discrete, linearly elastic springs related by an incompressible "shear layer" which is defined as a layer of linear-elastic material of unit thickness that resists vertical shear forces only [6]. Thus it is a refinement of the well known Winkler model which suffers from not describing shear influence. The use of a two parameter model to characterize the soil's response under loading can appear a too simplified concept. However, such a 
simplification seems coherent if one takes into account the variability and uncertainties related to the characterization of the soil. The spatial correlations which will be introduced further will ensure, in fact, a coherence of displacements, like it exists in a continuous medium. One should note that the two coefficients $k_{w}$ and $k_{s}$ are not soil-specific parameters. They are also affected by the rigidity of the pipe. In fact, these stiffness parameters depend on several factors, such as the length and/or the width (or diameter) of the pipe, the laying depth, the type of material used, and the type of the pipe bed. The value of these coefficients can only be approached by semi-empirical methods. For a same set of values, a parametric study, conducted in [7], illustrated the fact that $k_{w}$ varies from 1 to 3 . To the best of our knowledge the literature has not given ways on how to identify $k_{s}$ in practice from physical and geometrical data [8]. In order to integer the damping of the system, the Pasternak model is adapted and expanded to a Kelvin-Voigt model by adding dashpots. The stiffness matrix of the soil-pipe system has been derived in [9] by using the energy method. We will consider two relative soil-pipe stiffness ratios, $r_{p}$ and $r_{k s}$ which are defined by (3) and (4) and can be considered as governing parameters:

$$
\begin{aligned}
& r_{p}=\frac{1}{L} \sqrt[5]{\frac{E_{p} I}{k_{w}}} \\
& r_{k s}=\frac{k_{s}}{k_{w} D_{\text {ext }}^{2}}
\end{aligned}
$$

where $E_{p}, I, D_{\text {ext }}$, and $L$ and are respectively the pipe's Young modulus, the pipe's moment of inertia, the exterior diameter, and the length of the pipe.

\section{B. Joint-connection Stifness}

Sealing between pipes is ensured by joints made of cement mortar or more frequently of elastomer. The rigidity of these joints is as variable as the technologies and geometries employed: it can be very weak (flexible joints) or very high (welded joints). It is difficult to identify realistic numerical values of the joint stiffness even if some laboratory experiments have focused on this question [10]. In this work, we assume a continuity of vertical displacements at joint connection and contrary to a model of continuous beam, a model was developed which enables introducing discontinuities of rotation between the ends of the pipes. The joints between two adjacent pipes are assimilated to rotation springs with stiffness $R_{j}$ relating to the proportionality between the bending moment $M$ applied to the joint and the variation of the angle of rotation $\Delta \varphi$ :

$$
M=R_{j} \cdot \Delta \varphi
$$

To take into account this joint stiffness, the pipe-joint stiffness ratio $r_{\text {joint }}$ is introduced which is defined by:

$$
r_{\text {joint }}=\frac{R_{j} L}{E_{p} I}
$$

To summarize, the soil-pipe interaction system, one can say that it is governed by a geometric parameter $L$ (pipe's length) and three relative (dimensionless) stiffness ratio parameters: $r_{k s}$ (compression to shear), $r_{p}$ (soil to pipe), and $r_{\text {joint }}$ (connection to pipe).

\section{MODELING THE SPATIAL VARIABILITY OF SOIL AND ITS EFFECTS:THE CORRELATION LENGTH}

In many common geotechnical problems, the variability of soil is only one among many sources of uncertainty (others are for example reduced sampling measurement or model errors). It can be accounted for by taking conservative values of the soil parameters, even if geotechnicians need long practice to be able to justify the choice of these values [11]. In fact, the variability of soils cannot be reduced to case-by-case variability - as a result of its natural or man-made fabric (deposit processes, compaction processes). The soil properties can be considered as spatially structured. Thus, tools like autocorrelation functions or semi-variograms appear to be appropriate for modeling. One must then identify the standard deviation or coefficient of variation of the studied property as well as the correlation length $l_{c}$ (i.e. the distance above which the local properties at two points can be assumed to be independent). The first consequence of the spatial correlation is that the representative value of any soil property depends on the volume concerned by the problem to be solved. This question has been analyzed in detail during the drafting of Eurocode-7 but the code writers have limited themselves to general considerations, without prescribing any formal method: the representative value is only said to be a characteristic value defined as a cautious estimate of the parameter governing the studied limit state [12]. Nevertheless, accounting for spatial correlation has direct consequences on the safety of designs. A simple illustration of the effects of spatial variability is that of the rotation (tilting) of a foundation of length $L$ resting on a heterogeneous elastic soil and supporting a uniform loading. It was shown [13] that the magnitude of the rotation depends on the correlation length in the horizontal direction: it tends towards zero when $l_{c}$ is very small (which corresponds to very quickly varying properties, thus homogeneous at $L$ scale) or very large (which corresponds to very slowly varying properties, thus also homogeneous at $L$ scale) and it is maximum for an intermediate range. This illustrates a consequence of the spatially correlated variation of soil properties: tilting occurs only when the soil is not homogeneous and its magnitude depends both on the scatter in the soil properties (linearly) and on the correlation length, with a "worst case" for a particular range of $l_{c}$ values. Since the role of the longitudinal variability of the filling appears essential, we chose to model it by using the theory of the local average of a random field developed in [14]. The random field of the coefficients $k_{w}$ or $k_{s}$ is defined by three properties: its average value $\overline{k_{w}}\left(\right.$ resp. $\overline{k_{s}}$ ), its variance ${\overline{k_{w}}}^{2}$ (resp. ${\overline{k_{s}}}^{2}$ ) and its scale (or length) of correlation $l_{c}$ (resp. $l_{c s}$ ). These scales are related to a function of correlation $\rho(\tau)$ in (7) where $\tau$ points out the distance between two points, and which describes the spatial structure of correlation of the properties: $\rho(\tau)$ differs whether the properties vary more or less quickly while deviating from a given point. This correlation length (length from which the correlation between soil properties tend to disappear) depends on the characteristic (modulus, porosity, water content, etc.) and on the direction (horizontal or vertical). 


$$
\rho(\tau)=\exp \left(-2 \frac{|\tau|}{l_{c}}\right) \text { for } \tau \leq l_{c}
$$

The soil is subdivided in several zones. The random field value in each zone is thus a random variable whose value is estimated by the average of the space field over the zone. The local average and the variance in zone $i$ of length $D_{i}$ fulfil (8):

$$
\begin{gathered}
E\left[k_{w}\left(D_{i}\right)\right]=\overline{k_{w}} \\
\operatorname{Var}\left[k_{w}\left(D_{i}\right)\right]={\overline{\overline{k_{w}}}}^{2} \gamma\left(D_{i}\right)
\end{gathered}
$$

Equation (8b) shows that the local variance $\operatorname{Var}\left[k_{w}\left(D_{i}\right)\right]$ depends on the length $D_{i}$ of zone $i$ while following a variance reduction function $\gamma\left(D_{i}\right)$.

$$
\gamma\left(D_{i}\right)=2\left(\frac{l_{c}}{D_{i}}\right)^{2}\left(\frac{D_{i}}{l_{c}}-1+\exp \left(-\frac{D_{i}}{l_{c}}\right)\right)
$$

$\gamma\left(D_{i}\right)$ is a measure of the variance reduction due to the averaging of the random process according to the length of the studied zone. In this work, $k_{w}$ and $k_{s}$ follow a lognormal distribution. In order to show the effect of both the mean values of the Winkler and shear coefficients and their correlation length, six cases were considered (Table I) and applied to an example of a sewer section (set defined between two manholes) and which will be presented in further detail. The section is made of 20 pipes of unit length $L=3 \mathrm{~m}$. For each computation, the outputs which are linked to limit states are processed all along the section and their worst value is kept. Finally the Cumulative Density Function (CDF) of these parameters (stress, displacement, bending moment) is drawn and used for comparison. Figure 1 shows the CDF of the maximum bending stress in a concrete sewer's section.

TABLE I. VALUES OF $k_{w}, k_{s}, l_{c}$ AND $l_{c s}$

\begin{tabular}{|c|c|c|c|c|}
\hline parameters & $\boldsymbol{k}_{\boldsymbol{w}}\left(\mathbf{K N} / \mathbf{m}^{\mathbf{3}}\right)$ & $\boldsymbol{l}_{\boldsymbol{c}}(\mathbf{m})$ & $\boldsymbol{k s} \mathbf{( K N} / \mathbf{m})$ & $\boldsymbol{l}_{\boldsymbol{c s}}(\mathbf{m})$ \\
\hline Case A & 10 & 3 & 1 & 30 \\
\hline Case B & 10 & 30 & 1 & 3 \\
\hline Case C & 10 & 3 & 1 & 3 \\
\hline Case D & 10 & 3 & 10 & 3 \\
\hline Case E & 10 & 30 & 10 & 3 \\
\hline Case F & 10 & 3 & 10 & 30 \\
\hline
\end{tabular}

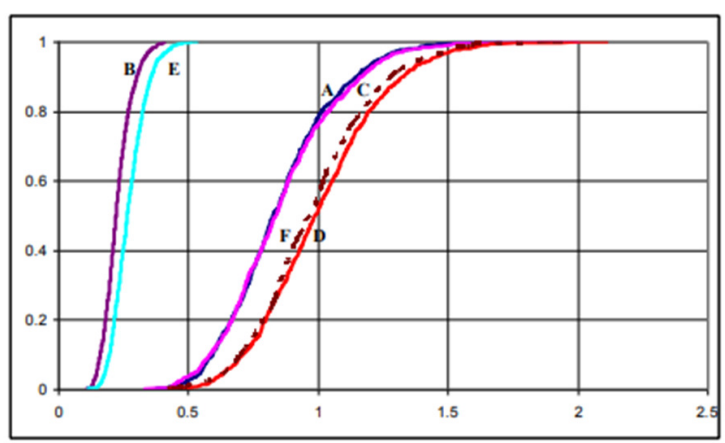

Fig. 1. CDF bending stress for the 6 different cases presented in Table I.

Two observations can be made:

- Not taking into account the shear effect is not conservative (case $\mathrm{C}$ compared to case $\mathrm{F}$ ) being given that the mean stress varies more than $20 \%$,
- The correlation length of the Winkler coefficient seems to have more importance than the correlation length of the shear coefficient (case B compared to $\mathrm{A}$ and $\mathrm{C}$ cases or case E compared to D and F cases).

\section{EQUATIONS OF MOTION}

The soil resistance to the pipe motion is generated by the relative motion $u$ between the pipe and the soil. The pipe resistance comes from the absolute displacement $U$ (Figure 2). The governing equation of the system is [15]:

$$
\begin{gathered}
{\left[M_{p}+M_{\text {sol }}\right]\{\ddot{U}\}+\left[C_{p}+C_{\text {sol }}\right]\{\dot{U}\}+\left[K_{p}+K_{w}+K_{s}\right]\{U\}=} \\
{\left[M_{\text {sol }}\right]\left\{\ddot{u}_{S}\right\}+\left[C_{\text {sol }} \dot{u}_{S}\right]+\left[K_{p}+K_{s}\right]\{u\}}
\end{gathered}
$$

in which $M_{p}$ and $M_{\text {soil }}$ are respectively the mass matrix of the pipe and of the soil, $K_{p}, K_{w}$ and $K_{s}$ the stiffness matrices of the pipe and the soil, and $C_{p}$ and $C_{\text {soil }}$ the damping matrices of the pipe and of the soil respectively. We note that the damping matrices are constructed from the Rayleigh damping. $U, \dot{U}, \ddot{\mathrm{U}}$, are respectively the vectors of absolute displacement, velocity and acceleration, $\mathrm{u}, u, \dot{u}$, and $\ddot{u}$ are the vectors of relative displacement, velocity and acceleration, and $u_{s}, \dot{u}_{s}$, and $\ddot{u}_{s} \mathrm{u}_{\mathrm{s}}$ are the vectors of ground displacement, velocity, and acceleration of the soil. Denoting the total stiffness as $[K]=$ $\left[K_{p}\right]+\left[K_{w}\right]+\left[K_{s}\right]$, the total mass as $[M]=\left[M_{p}\right]+\left[M_{\text {soil }}\right]$, the total damping as $[C]=\left[C_{p}\right]+\left[C_{\text {soil }}\right]$, and the absolute displacement as $\{U\}=\left\{u_{s}\right\}+\{u\}$, the equations of motion in terms of the relative displacement can be rewritten as:

$$
\begin{array}{r}
{[M]\{\ddot{u}\}+[C]\{\dot{u}\}+\lceil K]\{u\}=} \\
-\left[M_{p}\right]\left\{\ddot{u}_{S}\right\}-\left[C_{p} \dot{u}_{S}\right]-\left\lceil K_{p}\right]\left\{u_{S}\right\}
\end{array}
$$

These equations of motion are solved for each time history of the ensemble of real earthquake records. The boundary conditions can be considered either as fixed ends or as free ends if we want to eliminate their effects.

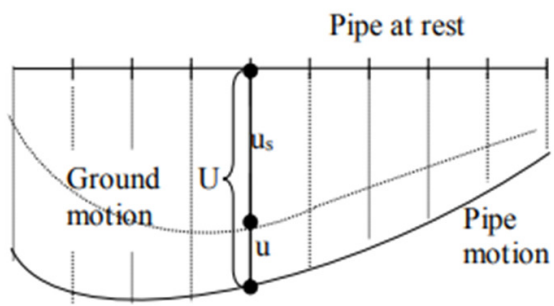

Fig. 2. Pipe and ground motion.

\section{PERFORMANCE FUNCTIONS AND LIMIT STATES}

Two performance functions are defined. One relates to the counterslope and corresponds to the SLS, by relating the sewer's hydraulic performance to the local counterslope. The presence of too high counterslopes harms the flow of the effluents and facilitates the clogging of the pipes by the sedimentation of the suspended particles. The corresponding limit state function is:

$$
G_{C S}=C S_{R}-C S_{S}
$$


where $C S_{R}$ is the maximum acceptable counterslope and $C S_{S}$ is the maximum simulated counterslope. The other performance function relates to the stresses and defines the equivalent of an ULS, by relating the cracking state (for a concrete pipe) to the bending stresses:

$$
G_{\sigma}=\sigma_{R}-\sigma_{S}
$$

where $\sigma_{R}$ is the yield tension stress of the concrete and $\sigma_{S}$ is the maximum bending stress. We assume that $C S_{S}$ and $\sigma_{S}$ also follow lognormal distributions, whose means and standard deviations are obtained from coupling Monte-Carlo simulations and finite element method, and that $C S_{R}$ as $\sigma_{R}$ also follow lognormal distributions of means and standard deviations of $4 \% \pm 0.8 \%$, and $2 \pm 0.3 \mathrm{MPa}$ respectively.

It should be noted that there is not any regulation limiting the counterslope, that's why we have chosen a $4 \%$ arbitrary limit based on the design considerations: trespassing such a value leads to damages or dismantling of the joints and to defects of sealing. The $\pm 0.8 \%$ can be seen as a model uncertainty on this SLS criterion. The target values of the reliability index to be reached are 3.8 for the ULS and 1.5 for the SLS with a service life of 50 years. In the following, we will consider two reliability indexes $\beta_{U L S}$ and $\beta_{S L S}$.

\section{CASE STUDY}

\section{A. Caracteristics of the Sewer's Section}

Lets consider a reinforced concrete material for the pipe $\left(E_{p}=25000 \mathrm{MPa}\right)$ with an exterior diameter $D_{\text {ext }}=1 \mathrm{~m}$, a thickness of $0.08 \mathrm{~m}$, made of a set of 20 pipes with $L=3 \mathrm{~m}$ unit length. It rests on a soil whose Winkler coefficient is $10 \mathrm{kN} / \mathrm{m}^{3}$ and shear coefficient is $10 \mathrm{kN} / \mathrm{m}$. This type of configuration corresponds to a pipe-soil ratio $r_{p}=0.678$. It must be noted that the pipe geometry intervenes in the expression of inertia $I$ and in that of the Winkler coefficients. The pipes are subjected to a deterministic uniform loading of $50 \mathrm{kN} / \mathrm{m}$ and to the earthquake of El-Asnam (Algeria, 1980). The ratio between the length of correlation $l_{c}$ and the pipe length $L$ is defined as $\lambda$. In the following sections are analyzed:

- the effect of the length of correlation on the response of the system,

- the effect of the relative soil-pipe stiffness, for an unfavorable length of correlation $(\lambda \# 1)$,

- and the effect of the relative joint-pipe stiffness.

The values of the obtained reliability $\beta$ indexes (ULS or SLS) must be considered rather comparatively that in an absolute way because of:

- the arbitrary character of the values retained for $C S_{R}$ as $\sigma_{R}$,

- the type of the probabilistic distribution,

- model uncertainties on soil characteristics $\left(k_{w}, k_{s}\right)$.

B. Effect of Correlation Length

The analysis is carried out for lengths of correlation ranging from $0.03 \mathrm{~m}$ to $1500 \mathrm{~m},\left(10^{-3}<\lambda<5 \times 10^{2}\right)$. Two types of joints are considered: rigid $\left(r_{\text {joint }}=10^{+5}\right)$ and flexible $\left(r_{\text {joint }}=10^{-5}\right)$.
Figures 3 and 4 show the ULS and SLS reliability indexes. It can be seen that there is a critical length of correlation and thus a critical value of $\lambda$ which causes the most unfavorable effects in the structure. The evolution of reliability is not monotonous. The existence of such a limit value is one of the invariants of the problems of (heterogeneous) soil-structure interaction [13]. Reliability indexes grow when $\lambda$ tends to zero or infinity. In these two situations, the soil tends to be homogeneous at the scale of the analysis, either because its variations are very fast are filtered by the pipe, or because its variations are very slow, inducing almost uniform values between close elements. We had seen that the rigid joints, and for a high coefficient of variation of $k_{w}$, do not satisfy the ULS for $\lambda$ between 0.01 and 7 , while the flexible joints provide much better results. On the other hand, if we consider the SLS, the flexible joints provide results a little worse than the rigid joints. One thus notes at this level a conflict in the choice of the "good pipe length" to answer the ULS and the SLS.

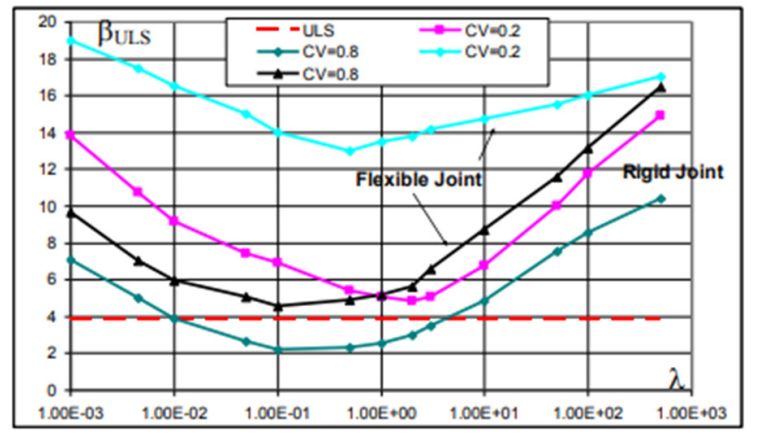

Fig. 3. Effect of the correlation length on the ultimate state reliability index for two values of the variation of coefficient $k_{w}$ and two of joints.

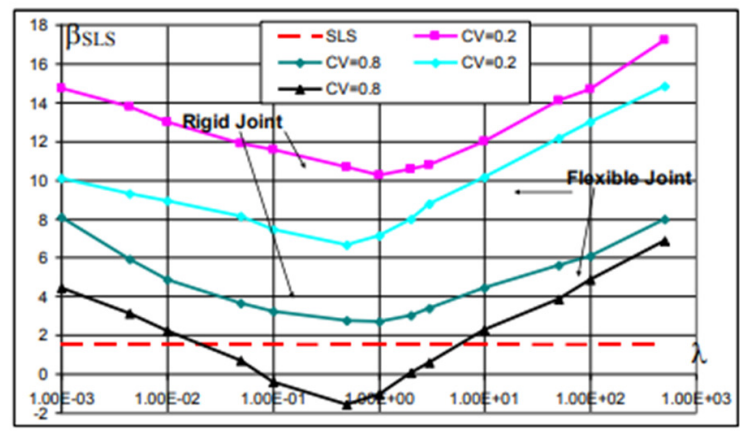

Fig. 4. Effect of the correlation length on the service limit state reliability index for two values of the variation of coefficient $k_{w}$ and two of joints.

\section{Effect of the Joint-pipe Stiffness Ratio}

The effect of joints on the behavior of the system was analyzed by varying the joint-pipe ratio $r_{\text {joint }}$ from $10^{-5}$ to $10^{+5}$. Figures 5 and 6 show the evolution of the reliability indexes for different coefficients of variation of Winkler coefficient. The strong influence of the joints' stiffness should be underlined since the rigid joints are penalizing with the ULS, by the fact that they necessarily induce more significant stresses. On the other hand, the flexible joints are penalizing with the SLS. An intermediate joint stiffness must be chosen in order to carry out 
the best compromise, since reliability is completely assured for both ULS and SLS. Finally, one can also note that for a given value of $r_{\text {joint }}$, the ULS index reliability and the SLS index reliability decrease with the increase of $k_{w}$ coefficients of variation.

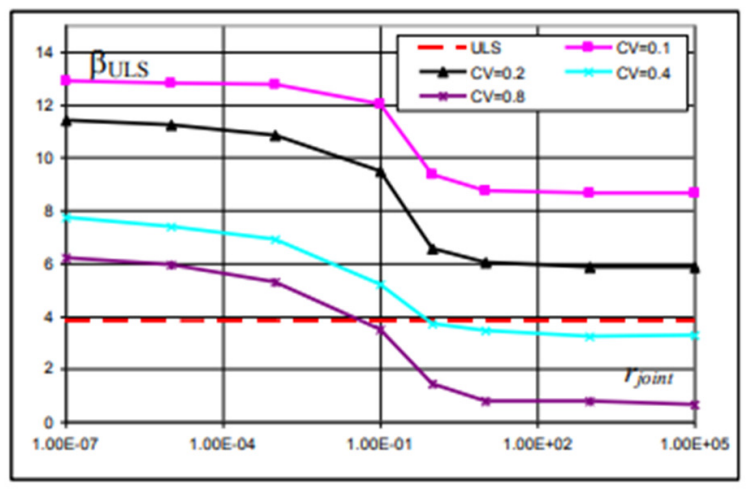

Fig. 5. Effect of joint-pipe stiffness ratio on ultimate limit state reliability index for four values of the variation of coefficient $k_{w}$.

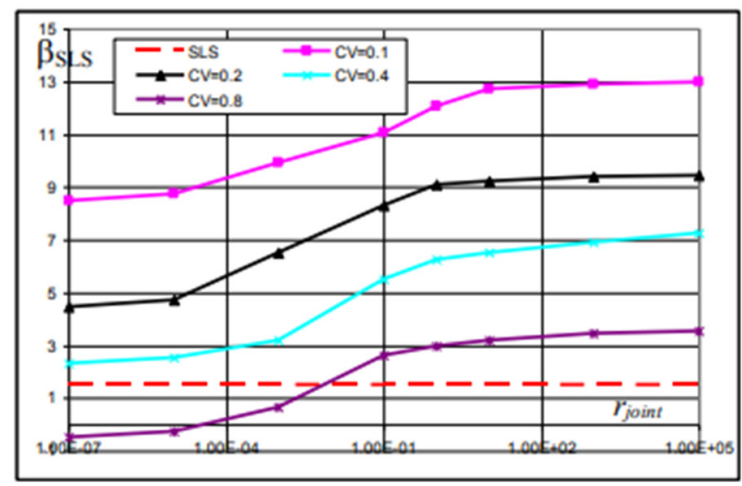

Fig. 6. Effect of joint-pipe stiffness ratio on the service limit state reliability index for four values of the variation of coefficient $k_{w}$

\section{Effect of the Soil-pipe Stiffness Ratio}

By maintaining the same set of pipes and soil parameters $\left(\lambda=1, r_{k s}=1\right)$, we vary the soil-pipe stiffness ratio $r_{p}$ in the range between 0.4 and 1.7 which corresponds to a Winkler coefficient in the range from $100 \mathrm{kPa}$ to $100 \mathrm{MPa}$ for four values of the coefficients of variation $\left(C V_{k w}=0.1,0.2,0.4\right.$, and 0.8$)$. While taking a length of correlation $l_{c}$ close to the length of the pipe $(\lambda \# 1)$, we refer to the situation in the vicinity of the most unfavorable geotechnical conditions. Figures 7 and 8 present the values of the reliability indexes. Whatever the type of joint, reliability is lower for high soil-pipe stiffness ratio, situation corresponding to sections of very stiff pipes or low soil stiffness. In the presence of rigid joints (Figure 7) and high coefficient of variation of the soil's parameter, the Eurocode $\beta_{U L S}$ is not respected (lower than 3.8). We can see that increasing the coefficient of variation induces reduction of the reliability of the pipe section. So, it is better to have flexible rather than rigid joints, it is better to privilege a lower $r_{p}$ to ensure the "good" reliability of the network.

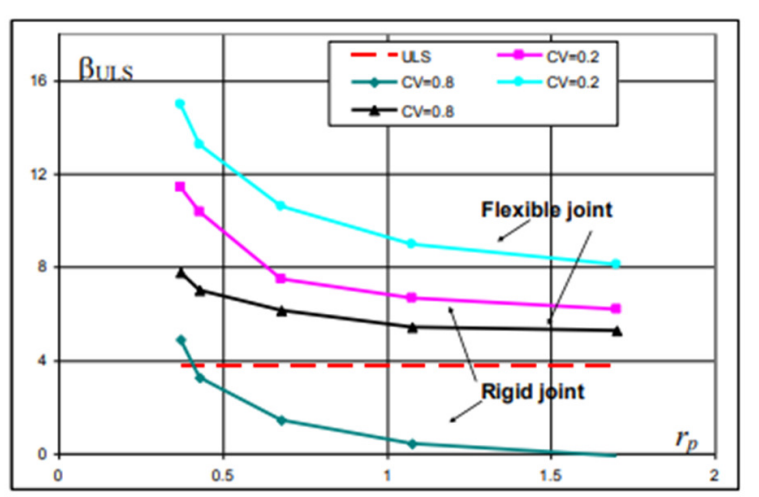

Fig. 7. Effect of soil-pipe stiffness ratio on the service ultimate state reliability index for four values of the variation of coefficient $k_{w}$.

In terms of counterslopes (Figure 8), it is better to have rigid than flexible joints. To ensure the reliability of the sewer's section using rigid joints imposes that the ground is rather homogeneous. It can be also noted that it is more profitable to be in the presence of a relatively homogeneous soil whose mechanical characteristics are poor that in the presence of a better ground but whose heterogeneity is more pronounced.

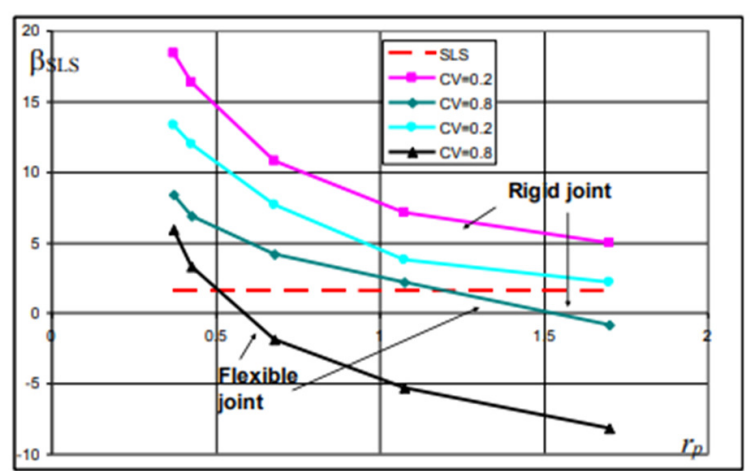

Fig. 8. Effect of soil-pipe stiffness ratio on the service limit state reliability index for four values of the variation of coefficient $k_{w}$.

\section{CONCLUSION}

A model which includes a description of the soil's spatial variability, within the frame of geostatistics has been developed in this paper. It is based on a mechanical description of the soil-structure interaction between a set of segmented buried pipes and the soil support represented by the Pasternak model. The need to take into account the soil spatial variability is clearly recognized. Several conclusions are drawn:

Soil heterogeneity induces effects (differential settlements, bending moments, stresses, and possible cracking) that cannot be predicted if homogeneity is assumed. These effects can significantly affect the SLS or ULS reliabilities. In addition to the effects of the soil stiffness, the magnitude of the induced stresses depends mainly on four factors or governing parameters: 
- The magnitude of the soil variability (i.e. its coefficient of variation).

- The soil-structure length ratio, which combines the soil fluctuation scale and a structural characteristic length (buried pipe length). A worst value, corresponding to the value leading (from a statistical point of view) to the (statistically) largest effects in the structure, can be found.

- The soil-structure stiffness ratio.

- The structure-connection (pipe-joint) stiffness ratio (relative flexibility).

The principal benefit of such an approach is that it provides some new approaches for better considering phenomena such as the geometrical irregularities in the longitudinal profile during the control of soil compaction of sewer trench filling. This kind of approach can also give the experts new tools for better calibration of safety in soil-structure interaction problems, when the soil variability is an influential parameter.

\section{REFERENCES}

[1] L. R. Khan and K. F. Tee, "Quantification and comparison of carbon emissions for flexible underground pipelines," Canadian Journal of Civil Engineering, vol. 42, no. 10, Jul. 2015, https://doi.org/10.1139/ cjce-2015-0156.

[2] M. J. O'Rourke and X. Jiu, Seismic Design of Buried and Offshore Pipelines. Buffalo, NY, USA: MCEER, University at Buffalo, State University of New York, 2012.

[3] Eurocode 7, Geotechnical design, Part 1: general rules. Brussels, Belgium: CEN, 1997.

[4] G. Manolis and D. E. Beskos, "Underground and lifeline structures," in Computer Analysis and Design of Earthquake Resistant Structures: A Handbook, vol. 3, D. E. Beskos and S. A. Anagnostopoulos, Eds. Southampton, UK: Computational Mechanics Publications, 1997, pp. 775-837.

[5] R. A. Hakim, M. S. A. Alama, and S. A. Ashour, "Seismic Assessment of an RC Building Using Pushover Analysis," Engineering, Technology \& Applied Science Research, vol. 4, no. 3, pp. 631-635, Jun. 2014, https://doi.org/10.48084/etasr.428.

[6] P. L. Pasternak, "On a new method of analysis of an elastic foundation by means of two foundation constants," in Gosudarstvennoe Izdatelstro Liberaturi po Stroitelstvui Arkhitekture, Moscow, Russia, 1954.

[7] S. M. Elachachi, M. Bensafi, M. Hamane, D. Nedjar, and D. Breysse, "Comportement longitudinal en flexion d'une conduite enterrée dans un remblai hétérogène," Revue Française de Géotechnique, no. 108, pp. 17-29, 2004, https://doi.org/10.1051/geotech/2004108017.

[8] K. Morfidis and I. E. Avramidis, "Formulation of a generalized beam element on a two-parameter elastic foundation with semi-rigid connections and rigid offsets," Computers \& Structures, vol. 80, no. 25, pp. 1919-1934, Sep. 2002, https://doi.org/10.1016/S0045-7949(02) 00226-2.

[9] S. M. Elachachi, D. Breysse, and L. Houy, "Longitudinal variability of soils and structural response of sewer networks," Computers and Geotechnics, vol. 31, no. 8, pp. 625-641, Dec. 2004, https://doi.org/ 10.1016/j.compgeo.2004.10.003.

[10] J. Buco, F. Emeriault, P. Le Gauffre, and R. Kastner, "Statistical and 3D Numerical Identification of Pipe and Bedding Characteristics Responsible for Longitudinal Behavior of Buried Pipe," in Pipeline Division Specialty Conference 2006, Chicago, IL, USA, Aug. 2006, https://doi.org/10.1061/40854(211)83.

[11] J. M. Duncan, "Factors of Safety and Reliability in Geotechnical Engineering," Journal of Geotechnical and Geoenvironmental Engineering, vol. 126, no. 4, pp. 307-316, Apr. 2000, https://doi.org/ 10.1061/(ASCE)1090-0241(2000) 126:4(307).
[12] J. B. Kovarik, "A propos des valeurs caractéristiques des propriétés des sols," in Journées ENPC de présentation des Eurocodes, 1996.

[13] D. Breysse, H. Niandou, S. Elachachi, and L. Houy, "A generic approach to soil-structure interaction considering the effects of soil heterogeneity," Géotechnique, vol. 55, no. 2, pp. 143-150, Mar. 2005, https://doi.org/10.1680/geot.2005.55.2.143.

[14] E. Vanmarcke, Random Fields: Analysis And Synthesis. Singapore, Hackensack, NJ, USA: Wspc, 2010.

[15] D. Nedjar, M. Hamane, M. Bensafi, S. M. Elachachi, and D. Breysse, "Seismic response analysis of pipes by a probabilistic approach," Soil Dynamics and Earthquake Engineering, vol. 27, no. 2, pp. 111-115, Feb. 2007, https://doi.org/10.1016/j.soildyn.2006.06.001. 